\title{
Medical Image of the Week: Pembrolizumab-induced Pneumonitis
}

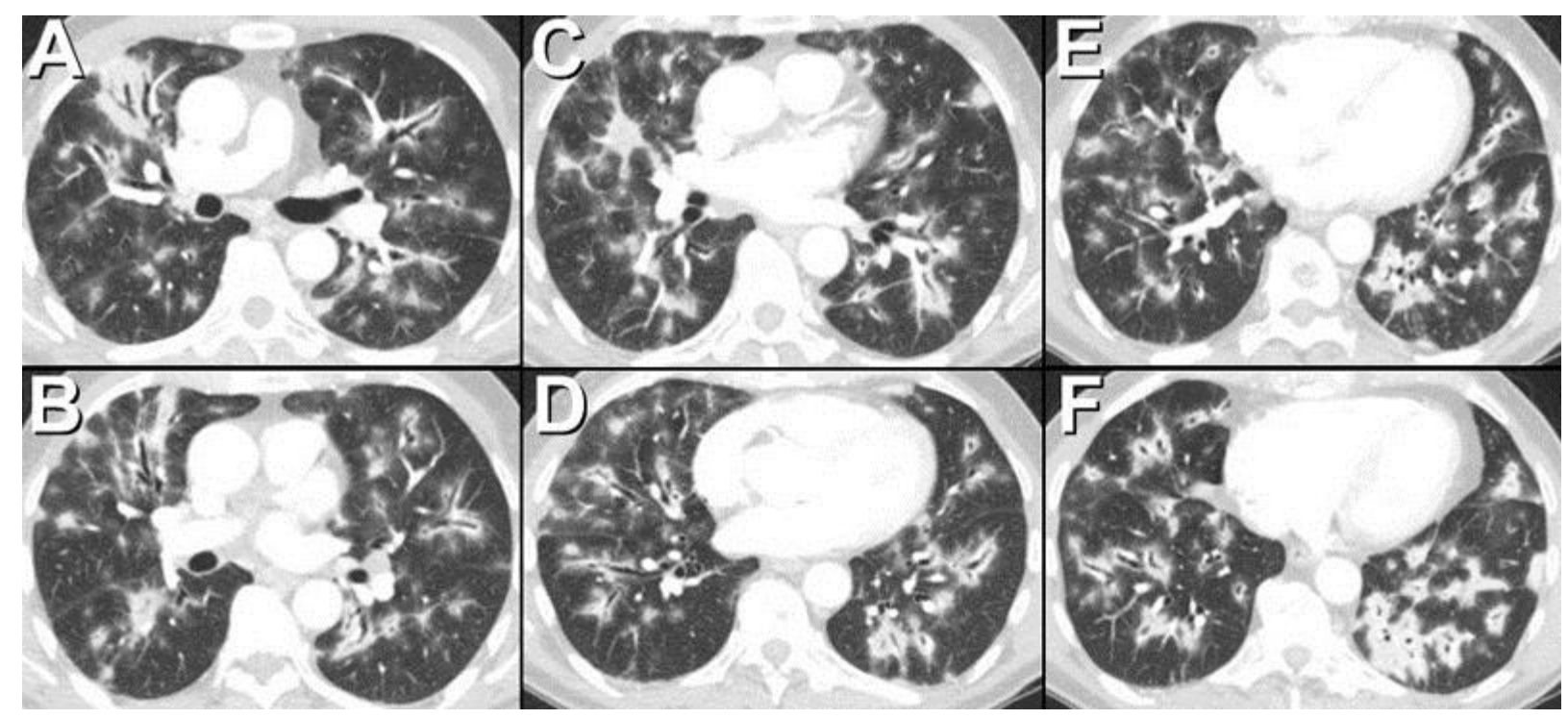

Figure 1. Thoracic CT showing multifocal, bilateral extensive lung opacities consistent with consolidation with a striking peribronchovascular distribution. Note the opacities are primarily distributed along the airways. Peripheral lung opacity, both ground-glass opacity and consolidation, is also present.

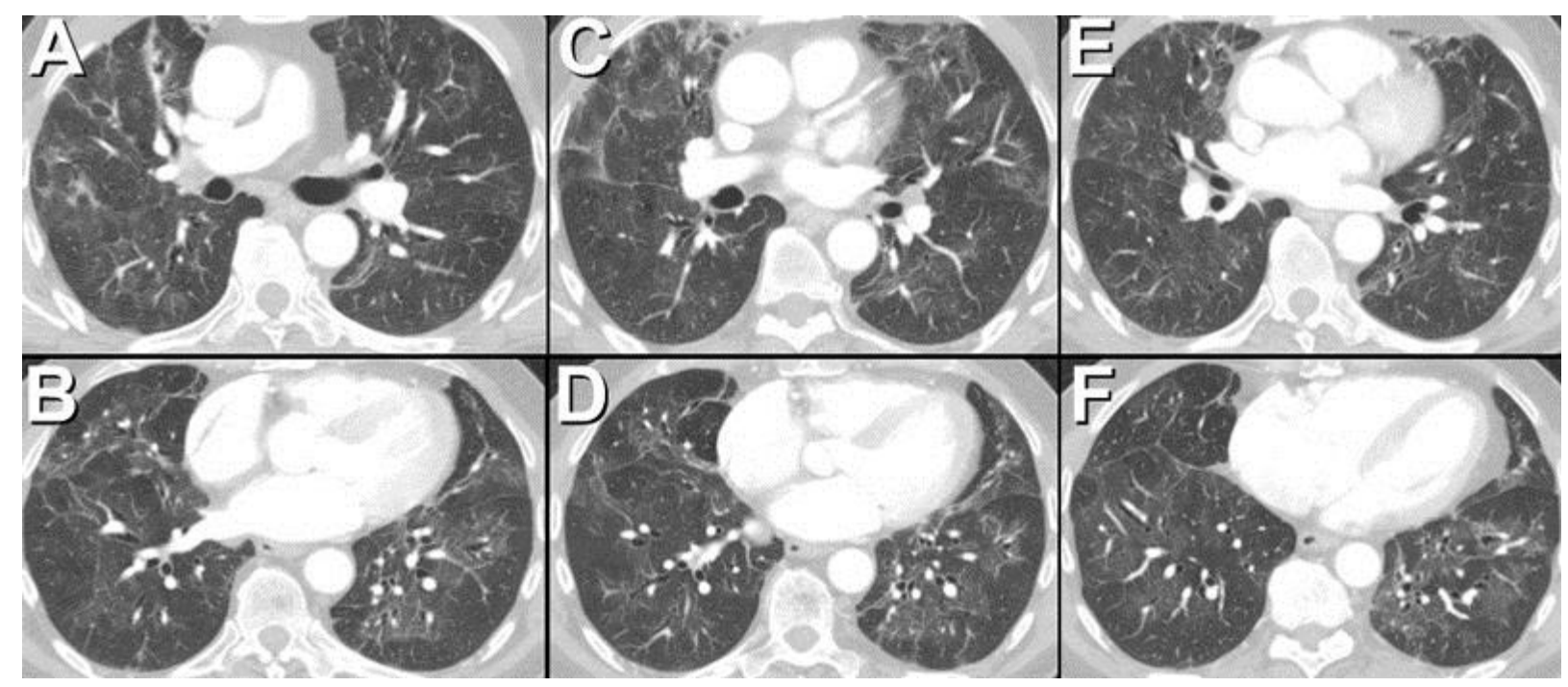

Figure 2. Axial thoracic CT performed several months after Figure 1 following discontinuation of the pembrolizumab and initiation, and subsequent tapering, of corticosteroid therapy, shows significant regression in the previously noted extensive peribronchovascular consolidation. Residual areas of consolidation and ground-glass opacity associated with architectural distortion are consistent with scarring. 
A 76-year-old man with metastatic melanoma, undergoing treatment with pembrolizumab, an antibody against programmed cell death 1 (PD-1), beginning 8 months ago developed low-grade fever, non-productive cough, and shortness of breath. A thoracic CT scan showed multifocal, bilateral extensive lung opacities (Figure 1). The patient underwent bronchoscopy with bronchoalveolar lavage which showed nonspecific inflammatory changes associated with foci of organizing pneumonia. Microbiologic studies, including Coccioides antibody enzyme immunoassay and Aspergillus antigen, were negative.

The patient was begun on corticosteroid therapy for presumed medication-induced pulmonary injury, manifestation as an organizing pneumonia pattern, due to pembrolizumab. Over the ensuing months, his symptoms abated and his CT scan abnormalities regressed (Figure 2).

Organizing pneumonia may occur as an idiopathic, primary pulmonary process, often referred to as "cryptogenic organizing pneumonia," or may occur in the context of a number of systemic conditions, a situation often referred to as secondary organizing pneumonia. Among the various etiologies of secondary organizing pneumonia, medication-induced pulmonary injury is fairly common and when imaging features of organizing pneumonia are seen, careful correlation regarding the possibility of a medication-induced etiology should be undertaken. Recently, three cases of pembrolizumab-induced pneumonitis were described, two being consistent with organizing pneumonia (1).

The thoracic CT findings of organizing pneumonia include peripheral and peribronchovascular consolidation and ground-glass opacity, areas of consolidation surrounding ground-glass opacity (often referred to as the "atoll" or reverse groundglass halo" sign- see Medical Image of the Week: The Atoll Sign in Cryptogenic Organizing Pneumonia), single and multiple nodules, and perilobular consolidation. The case illustrates a dramatically peribronchovascular distribution of pulmonary consolidation as a manifestation of medication-induced organizing pneumonia.

Michael B. Gotway, MD

Department of Radiology

Mayo Clinic Arizona

Scottsdale, AZ USA

\section{Reference}

1. Leroy V, Templier C, Faivre JB, Scherpereel A, Fournier C, Mortier L, WemeauStervinou L. Pembrolizumab-induced pneumonitis. ERJ Open Res. 2017 May 2;3(2). pii: 00081-2016. [CrossRef] [PubMed] 